\title{
Best Practices and Tools for Titrating Basal Insulins: Expert Opinion from an Indian Panel via the Modified Delphi Consensus Method
}

\author{
Sunil M. Jain · Krishna Seshadri · A. G. Unnikrishnan • Manoj Chawla • \\ Pramila Kalra · V. P. Vipin · E. Ravishankar · Jay Chordia · Sambit Das • \\ Jasjeet Wasir · S. M. Bandookwala · Neelakshi Deka · Ghanshyam Agarwal • \\ G. Vijaykumar · Suhas Erande
}

Received: November 20, 2019 / Published online: February 3, 2020

(C) The Author(s) 2020

\section{ABSTRACT}

Aim: To develop an evidence-based expert group consensus document on the best practices and simple tools for titrating basal insulins in persons with type 2 diabetes mellitus (T2DM).

Background: Glycemic control is suboptimal in a large proportion of persons with T2DM, despite insulin therapy, thereby increasing the risk of potentially severe complications. Early

Enhanced Digital Features To view enhanced digital features for this article go to: https://doi.org/10.6084/ m9.figshare.11604675.

S. M. Jain $(\bowtie)$

TOTALL Diabetes and Hormone Institute, Indore,

Madhya Pradesh, India

e-mail: sunilmjain@gmail.com

K. Seshadri

Apollo Specialty Hospitals, Chennai, Tamil Nadu, India

A. G. Unnikrishnan

Chellaram Diabetes Institute, Pune, Maharashtra, India

\section{Chawla}

Lina Diabetes Care and Mumbai Diabetes Research

Centre, Mumbai, Maharashtra, India

P. Kalra

M S Ramaiah Memorial Hospital, Bangalore,

Karnataka, India initiation of insulin therapy and appropriate dose titration are crucial to achieving glycemic targets. Attitudes and practices among healthcare professionals (HCPs) and perceptions about insulin therapy among persons with diabetes contribute largely to suboptimal glycemic control. Improving HCP-patient communication, encouraging the use of additional educational tools, and providing support for the titration process to increase confidence, both at the initiation visit and at home, facilitate the optimization of dose titration. In Indian settings, specific guidelines and a consensus statement are lacking on the optimal insulin initiation dose, frequency of dose titration, and basal insulin profile needed to achieve optimal

\author{
V. P. Vipin \\ Aster Medcity, Cochin, Kerala, India \\ E. Ravishankar \\ Apollo Hospitals, Hyderabad, Telangana, India \\ J. Chordia \\ Fortis JK Hospital, Udaipur, Rajasthan, India \\ S. Das \\ Endeavor Clinic-Center of Diabetes and \\ Endocrinology, Bhubaneswar, Odisha, India \\ J. Wasir \\ Medanta-The Medicity, Gurgaon, Haryana, India \\ S. M. Bandookwala \\ Lilavati Hospital and Research Centre, Mumbai, \\ Maharashtra, India
}


titration. In clinical practice, physicians and persons with diabetes often do not adhere to the titration algorithms that currently exist for the purpose of achieving optimal titration as they perceive these to be very cumbersome. In this context, a group of experts met at an advisory board meeting and arrived at a consensus on best practices for the titration of basal insulin in persons withT2DM in India, using the modified Delphi methodology.

Review Results: After a review of evidence and further discussions, the expert group provided recommendations on insulin initiation dose, ideal period for titration in practice, titration regimen for use in practice, basal insulin profile for titration, and choosing a self-monitoring blood glucose schedule for titration.

Conclusions: In the management of T2DM, insulin can be effectively titrated by following a few simple recommendations. The use of second-generation basal insulin aids in mitigating the risk of hypoglycemic events. The implementation of a simplified titration regimen is crucial to achieving glycemic targets and longterm treatment goals.

Keywords: Basal insulin; Glargine-U300; Hypoglycemia; Insulin initiation; Secondgeneration basal insulins; Self-monitoring of blood glucose; Titration

N. Deka

Apollo Hospitals, Guwahati, Assam, India

G. Agarwal

R.P. Diabetic Endocrine Center, Lucknow, Uttar Pradesh, India

G. Vijaykumar

Diabetes Medicare Center, Chennai, Tamil Nadu, India

S. Erande

Akshay Hospital and Diabetic Speciality Centre and Insulin Pump Centre, Pune, Maharashtra, India

\section{Key Summary Points}

Glycemic control is suboptimal in a large proportion of persons with type 2 diabetes mellitus (T2DM), despite insulin therapy, thereby increasing the risk of potentially severe complications. Early initiation of insulin therapy and appropriate dose titration are crucial to achieving glycemic targets.

In clinical practice, physicians and persons with diabetes often do not adhere to the titration algorithms that do exist for the purpose of achieving optimal titration as they perceive these to be very cumbersome. In this context, a group of experts met at an advisory board meeting and arrived at a consensus on best practices for the titration of basal insulin in persons with T2DM in India, using the modified Delphi methodology.

In the management of DM, insulin can be effectively titrated by following a few simple recommendations. The use of second-generation basal insulin aids in mitigatiing the risk of hypoglycemic events. The implementation of a simplified titration regimen is crucial to achieving glycemic targets and long-term treatment goals.

\section{INTRODUCTION}

Type 2 diabetes mellitus (T2DM) is a chronic, progressive disorder, associated with multiple pathophysiologic abnormalities. Insulin resistance and reduced insulin secretion due to progressive beta-cell dysfunction represent the two key pathophysiological defects in T2DM. Evidence suggests a depletion of $>50 \%$ of the beta cells at the time of T2DM diagnosis, and continuation of the loss and disease progression are the major causes of concern. Therefore, insulin therapy for the management of T2DM is 
crucial to supplement the endogenous insulin that is inadequately produced [1].

However, in clinical practice, glycemic control remains suboptimal despite the initiation of insulin therapy, possibly due to therapeutic or clinical inertia. Resistance to insulin therapy can occur at the stages of initiation, titration, and intensification of therapy, and both patient- and physician-related factors act as major barriers to the achievement of optimal glycemic control. Tackling therapeutic inertia with appropriate strategies is a crucial step towards improving long-term outcomes [2].

Titration is a key element of the therapeutic regimen required by persons with $\mathrm{T} 2 \mathrm{DM}$ to achieve euglycemia on insulin. In Asian countries, such as India, the average titration dose of basal insulin remains 18 units in practice; in contrast, in clinical trials, titration of doses up to 40 units has been reported. Insulin once initiated is most often not adequately titrated, which is an unmet need in the optimization of glycemic control. This implies that proper titration is overlooked in India due to several physician-related and person-related factors. In this context, a group of experts executed a plan to conduct a steering meeting followed by an advisory board meeting, with the aim to develop a consensus on the achievement of optimal titration with basal insulin by using the modified Delphi methodology.

\section{METHODOLOGY}

The modified Delphi consensus methodology was considered to arrive at a consensus, as the key opinions of many experts were needed on the topic of discussion. Prior to the steering committee meeting, topics relevant to key challenges to adequate titration were selected. The topics of discussion included initial insulin dose, titration inertia, timing of titration, methods to titrate insulin doses, ideal insulins for titration, and provider- versus physician-led titration. Appropriate questions on each of these topics were developed. During the steering committee meeting, these questions were discussed, and a framework of the different phases of the project was planned. The steering committee recommended a simple empowerment algorithm to break titration inertia: a once-weekly titration strategy that included uptitration by 2 units until fasting plasma glucose (FPG) levels reached between 120 and $140 \mathrm{mg} /$ $\mathrm{dL}$ and down-titration by 2 units to where the FPG levels would be $<90 \mathrm{mg} / \mathrm{dL}$. The first follow-up visit would be after 2 weeks of the initiation of insulin therapy. The committee suggested including the self-management of blood glucose as an important component of discussion. The other suggestions from the committee included emphasis on patient empowerment and management, defining insulin profiles for titration, and re-designing of the Delphi questionnaire.

The questionnaires were appropriately revised and subsequently emailed to all of the participating experts. Agreement or disagreement to each of the questions was sought. The questions that were agreed upon by $>80 \%$ of respondents were considered to be expert panel recommendations; those questions that did not receive consensus were subsequently discussed and debated during the advisory board meeting. This document captures the key elements of both these meetings.

This article is based on previously conducted studies and does not contain any studies with human participants or animals performed by any of the authors.

\section{INSULIN INITIATION AND INITIAL DOSE IN PERSONS WITH T2DM}

In clinical practice, insulin therapy is initiated very late in the course of the disease, following failure of the patient to achieve glycemic goals with multiple oral hypoglycemic agents and when blood glucose values are well above the recommended targets [1]. Evidence suggests that in real-world settings in Asia, mean glycated hemoglobin $\left(\mathrm{HbA}_{1 \mathrm{c}}\right)$ levels are $\geq 9 \%$ and mean diabetes duration is 9.3 years at the time of initiation of insulin therapy with basal insulin [3]. Therefore, it is crucial to tackle the persistent barriers that contribute to delays in the initiation of insulin therapy for the management of diabetes mellitus. Complicated 
regimens and unrealistic goals can hamper the patient's involvement and trust in the treatment pathway. A simple and practical therapeutic approach helps in patient empowerment, provides the context for targeting the goals in the management of diabetes mellitus, and boosts the confidence of the persons facing these barriers [1].

Basal insulin therapy typically starts at a low dose (8-10 units) to be taken at bedtime, with the aim of targeting morning fasting glucose. In numerous key clinical trials of basal insulin, the initial initiation dose of basal insulin used was 10 units/day [3].

Key Recommendations from Experts on Insulin Initiation Dose

A dose of 8-12 units of basal insulin is the ideal initiation dose

When hypoglycemia is a concern, a dose of 4-6 units may also be considered

Persons with diabetes should be apprised of the need for insulin titration at the initiation of insulin therapy so that they are prepared for the procedure. They should be appropriately educated that increasing insulin doses does not necessarily mean their condition has deteriorated, and this message should be consistently reinforced. The titration schedule should be captured in the prescription where possible

Persons with diabetes should be requested to visit their treating healthcare professional within 1-2 weeks after the initiation of insulin therapy to start the titration process

\section{IMPORTANCE OF SELF- MONITORING OF BLOOD GLUCOSE FOLLOWING INITIATION OF INSULIN THERAPY}

Self-monitoring of blood glucose (SMBG) constitutes one of the key tools for the management of diabetes mellitus [4]. The selfmonitoring of glucose levels encourages, empowers, and enables persons with diabetes to understand the effects of lifestyle changes and medications on their blood glucose levels. It also allows persons with diabetes to implement the required interventions to help improve their glycemic control. Evidence suggests improved glycemic control in persons with T2DM who regularly use SMBG. Self-monitoring of the blood glucose levels also helps persons detect hypoglycemia and adjust their insulin dosage appropriately [5].

At the initiation visit, clear instructions should be given to persons on how to selfmonitor their blood glucose level. Simple and specific steps should be used to teach SMBG, based on each person's level of comprehension. Written recommendations for the frequency of testing and time of testing with desired results should be provided. The SMBG procedure should be taught at the initial visit and re-emphasized at follow-up visits [4].

Key Recommendations from Experts on Self-Monitoring of Blood Glucose Following Insulin Initiation

Following the initiation of insulin therapy, self-monitoring of blood glucose (SMBG) must be recommended 2-3 times a week for optimizing titration

\section{WHEN TO TITRATE BASAL INSULIN?}

\section{Insulin Titration is Defined as the First 8-12 Weeks After the Initiation of Insulin Therapy}

In a pooled analysis of selected data from 15 treat-to-target (TTT) randomized controlled studies, treatment outcomes in persons with T2DM who were inadequately controlled on oral antidiabetic drugs (OADs) were evaluated following the introduction of insulin glargine (IGlar). Early (0-12 weeks) and later (12-24 weeks) glycemic control and event rates of hypoglycemia were explored during the 24 -week treatment period. The analysis included 2837 persons who received IGlar was added as adjunct to metformin, to a sulfonylurea, or to both. An early and sustained improvement in glycemic control was noted in the persons under 
study following the addition of IGlar to the therapeutic regimen using TTT titration. Improvements in $\mathrm{HbA}_{1 \mathrm{c}}$ and FPG levels, noted at weeks 12 and 24 following the initiation of IGlar therapy, were similar in all treatment groups. One of the key observations reported in the analysis was the achievement of $>80 \%$ of the maximum treatment effect in terms of reductions in $\mathrm{HbA}_{1 \mathrm{c}}$ at 12 weeks of basal insulin therapy [6].

Key Recommendations for Ideal Period for Titration in Practice (When to Titrate Basal Insulin?)

Active titration period is defined as the period when the physician is adjusting the dose of basal insulin. This period typically lasts for up to 12 weeks after the initiation of insulin therapy

The panel agreed that the maximal decrease in glycated hemoglobin $\left(\mathrm{HbA}_{1 \mathrm{c}}\right)$ and fasting plasma glucose (FPG) goals are to be achieved by week 12 after the initiation of insulin therapy and that the minimal reduction is to be seen after 12 weeks

\section{INERTIA ABOUT INSULIN TITRATION: AN ONGOING CHALLENGE FOR PHYSICIANS AND PERSONS WITH T2DM}

Titration inertia is multifactorial and can be a result of reluctance/inaction by the healthcare professional (HCP), person with diabetes, or both. Person-related factors, such as fear of hypoglycemia and weight gain, can result in under-titration. Concerns about the impact of insulin therapy on their quality of life may impede insulin use by persons with T2DM. Lack of flexibility and difficult-to-follow titration algorithms constitute a major cause of concern in persons with diabetes. Irregularity in SMBG, lack of adequate healthcare resources and practitioners, and inadequate educational programs for persons regarding effective titration are other factors that lead to poor titration. Additionally, HCPs may fail to encourage aggressive titration due to reluctance or concerns expressed by persons with diabetes [2].

\section{TACKLING TITRATION INERTIA WITH SIMPLE TITRATION ALGORITHMS FOR EFFECTIVE OUTCOMES}

Simple and effective titration algorithms that can be individualized by the HCP based on clinical considerations are highly recommended and aid in tackling titration inertia [7]. Dose titration of IGlar has been tested in numerous clinical studies using different titration algorithms based on FPG values to allow flexible dose adjustments to persons and meet their insulin needs. The studies include the INSIGHT (Implementing New Strategies with Insulin Glargine for Hyperglycemia Treatment) study [8], the LANMET (LANtus plus METformin) study [9], the AT.LANTUS (A Trial comparing LANTUS Algorithms to achieve Normal blood glucose Targets in subjects with Uncontrolled blood Sugar) study [10], and a TTT trial [11]; dose adaptations varied from 1 to 8 units in these trials.

\section{KEY INFORMATION REGARDING BASAL INSULIN TITRATION SHOULD BE REINFORCED AT REGULAR INTERVALS}

A timely proactive follow-up is a critical factor to achieving success in terms of effective titration and can be fulfilled by the HCP or other members of the healthcare team. The importance of basal insulin titration should be reinforced at each visit [7].

In an online survey conducted in the USA, France, and Germany, the attitudes of HCPs $(n=386)$ and persons withT2DM $(n=318)$ at the initiation of basal insulin therapy and titration were determined [1]. The survey reported that $>75 \%$ of HCPs discussed titration at the time of insulin initiation, while only $16-28 \%$ of persons with T2DM recalled such discussions. The survey also showed that nearly 
$32-42 \%$ of these persons were not aware of the importance and benefits of titration of basal insulin and that only $28-39 \%$ remembered the mention of duration of time required to attain the targeted glycemic goals [1].

At each visit, persons should be provided appropriate feedback on the glycemic levels they have achieved with insulin therapy. Such feedback helps the persons to understand the association between their blood glucose levels and the symptoms (e.g., tiredness, frequent urination, blurred vision, etc.). Follow-up visits following insulin initiation should be scheduled at 2 weeks, at the most. Pro-active contact with the persons receiving insulin therapy is critical to encourage them as well as to keep them motivated [1].

Ideally, insulin titration should be based primarily on blood glucose levels, but many other factors are also important. It should be noted that in many studies when blood glucose levels surpassed $180 \mathrm{mg} / \mathrm{dL}$, the insulin dose was increased by at least 6 units. However, the panel recommendations are based on the need to simplify the titration of insulin in the Indian context.

Key Recommendations for Titration Regimen in Practice (How to Titrate Basal Insulin?)

A once-weekly titration regimen should be adopted in which up-titration is by 2 units until the FPG levels are between 90 and $120 \mathrm{mg} / \mathrm{dL}$ and down-titration is by 2 units when the FPG levels are $<90 \mathrm{mg} / \mathrm{dL}$. This regimen may be a simple and effective method in the Indian context; where possible insulin dose can also be titrated 2 units every 3 days

The recommended maximum daily dose of basal insulin is 0.5 units $/ \mathrm{kg}$ body weight. The maximum average daily dose of basal insulin titration in the Indian context is 30 units although the panel did note that there is no strict maximum dose for basal insulin. This recommendation was based on the clinical experience of the expert panel

Person/caregiver injection techniques should be reviewed at the first visit and at every follow-up visit
Table e continued

Log books and mobile apps are useful tools to help the patient keep a record of the titration process

Once-a-month follow-up is recommended until optimal dose of basal insulin is achieved

Documenting episodes of hypoglycemia and maintaining a symptom diary may help persons with type 2 diabetes mellitus (T2DM) overcome the fear of hypoglycemia during the titration period

\section{PERSON EMPOWERMENT IN TITRATION: FROM CONCEPT TO PRACTICE}

The engagement and education of family members are very important aspects of the discussions on the titration algorithm with patients. Clearly written instructions to the patient and/or caregiver and provision of hypothetical scenarios of blood glucose values in the office help the patient to understand the practicality of titration. Simplified titration algorithms that are simple, safe, and effective and which can be customized and individualized should be considered. Simple algorithms and supportive technology can be used as alternatives for persons who find it difficult to calculate the dose required for titration [7].

\section{IMPORTANCE OF SMBG IN THE OPTIMIZATION OF TITRATION}

The desired frequency of SMBG testing for insulin dose adjustments is often debated in clinical practice. In persons with T2DM on bedtime neutral protamine Hagedorn $(\mathrm{NPH})$ insulin or only a basal insulin, only fasting state (i.e., before breakfast) SMBG is required. In persons taking more than one insulin, SMBG testing before each meal and before the bedtime snack is needed. The guideline-recommended frequency of SMBG testing is very unrealistic in practice. Therefore, a defined frequency of 
SMBG testing during titration and after achievement of the agreed-upon targets, which is more realistic and practical, is the need of the hour [12].

Key Recommendations for Choosing the Self-Monitoring of Blood Glucose Schedule for Titration

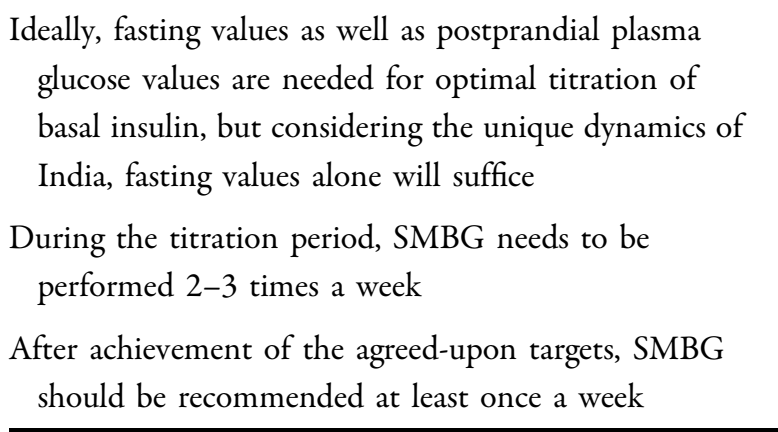

\section{PERSON-LED VERSUS PHYSICIAN- LED TITRATION ALGORITHMS}

Insulin titration can be performed either by the patient or the healthcare provider and team, based on the patient's ability and preparedness. Insulin titration is an important step that aids persons to achieve glycemic goals, and evidence suggests that persons can titrate insulin as effectively as HCPs.

Person-led titration algorithms have been found to be more effective in terms of the achievement of near-target glycemic levels. Several TTT trials of insulins have provided greater insights into the principle of patient self-titration. In the AT.LANTUS study [10], two TTT algorithms, one patient-led and one physician-led algorithm, respectively, for the titration of IGlar 100 units/mL (Gla-100) were compared. The results showed that in comparison to physician-led titration, a simple patientadministered titration algorithm significantly improved glycemic control, with a low incidence of severe hypoglycemia [10].

In another TTT trial, the multinational ATLAS (Asian Treat to Target Lantus Study) trial, the change in mean $\mathrm{HbA}_{1 \mathrm{c}}$ at week 24 was compared in the patient-led versus physicianled titration groups [13]. The authors noted that patient-led titration resulted in a significantly higher drop in $\mathrm{HbA}_{1 \mathrm{c}}$ at 24 weeks versus physician-led titration $(-1.40$ vs. $-1.25 \%$, respectively; mean difference $-0.15 ; 95 \%$ confidence interval -0.29 to $0.00 ; p=0.043)$. The mean decrease in fasting blood glucose was greatest in the patient-led group $(-2.85 \mathrm{vs}$. $-2.48 \mathrm{mmol} /$ $\mathrm{L} ; p=0.001)$. The authors of the study concluded that patient-led IGlar titration achieved near-target blood glucose levels in Asian persons with uncontrolled T2DM who were on two oral glucose-lowering drugs, demonstrating that Asian persons can self-up-titrate insulin dose effectively when given the appropriate support and guidance [13].

Reports from the INSIGHT study suggest that persons were able to titrate the insulin dose by 1 unit/day until reaching the targeted goal with the aid of a simple-to-use patient self-titration algorithm [14]. Similarly, several other key clinical trials have shown that glycemic targets were attained with the use of simpler persondirected titration algorithms (Table 1).

\section{DEVICE-SUPPORTED TITRATION FOR ACHIEVING GLYCEMIC GOALS}

The efficacy and safety of titrating basal insulin using device-supported applications has been assessed in persons with T2DM in several trials. Established basal insulin titration algorithms in the application guide the persons to the dosage of basal insulin they need to administer based on their blood glucose level. The applications also provide several self-tracking and streamlined communication tools, such as text messages and blood glucose readings, which can be automatically integrated into the application via wireless glucometers. The applications also offer daily reminders for blood glucose testing, alerts when blood glucose is low, and information on diabetes medications. The applications can be synchronized to allow clinicians to remotely monitor persons with diabetes [17]. 
Table 1 Simple person-led titration algorithms in key clinical trials

\begin{tabular}{ll}
\hline Study & Dose adaptation (patient-led titration algorithm) \\
\hline 2 units dose adaptation every 3 days & AT.LANTUS [10] \\
$2-4$ units dose adaptation every 3 days & LANMET [9] and INITIATE [15] \\
3 units dose adaptation every 3 days & PREDICTIVE 303 [16] \\
\hline
\end{tabular}

AT.LANTUS A Trial comparing Lantus Algorithms to achieve Normal glucose Targets in subjects with Uncontrolled blood Sugar, LANMET LANntus plus METformin, INITIATE INITiate Insulin by Aggressive Titration and Education, PREDICTIVE 303 Predictable Results and Experience in Diabetes through Intensification and Control to Target: An International Variability Evaluation 303

Key Recommendations for Provider versus Person-Led Titration in Practice

People empowerment plays an integral role in titration of insulin. However, physician assistance will be required during the titration process

Self-management should be promoted to persons with diabetes at all points of the insulin journey

Technology can play a role in helping optimize insulin therapy but may not be of much use in illiterate persons

\section{PROFILE OF BASAL INSULIN FOR TITRATION}

Insulin with low hypoglycemic risk and which allows for optimal titration once initiated is the need of the hour in clinical practice. Even mild hypoglycemic episodes can shake a patient's confidence. Evidence suggests that persons with T2DM reduce their insulin dose after a mild hypoglycemic episode.

Hypoglycemia during the first 3 months of basal insulin therapy is a predictor for long-term hypoglycemia risk. In one observational study, the authors conducted a retrospective analysis of the patient database of general practitioners' electronic medical records [18]. The study used medical records from five European countries and the USA. The aim of the study was to evaluate short-and long-term glycemic control and hypoglycemia incidence in insulin-naïve persons with T2DM who were initiating basal insulin therapy, with or without OADs. The total number of participants was 40,627. The authors noted that hypoglycemia during the initial 3-month period was associated with a long-term risk of these events over the ensuing 3 to 24 months. These results led to the conclusion that the effective titration of insulin doses that optimizes blood glucose control without increasing the risk of hypoglycemia is crucial [18].

\section{BASAL INSULIN VERSUS PREMIX FOR INSULIN TITRATION}

The INITIATE (INITiate Insulin by Aggressive Titration and Education) study was a 28 -week, randomized, multicenter, open-label, parallelgroup study that assessed the safety of twicedaily biphasic insulin aspart 70/30 (BIAsp 70/30) with once-daily IGlar in 233 insulinnaïve persons with T2DM [19]. The results showed a significantly greater incidence of minor hypoglycemic events in the BIAsp 70/30 group versus the IGlar group $(p<0.05)$. Similarly, minor hypoglycemia was reported by $43 \%$ of persons in the BIAsp 70/30 group and by only $16 \%$ in the IGlar group $(p<0.05)$ [19].

The LAPTOP trial was a 24 -week, randomized, parallel-group trial that assessed the safety of twice-daily premixed insulin (70:30 NPH) with once-daily IGlar plus OADs in 371 persons withT2DM inadequately controlled on OADs [20]. A significantly greater number of confirmed hypoglycemic episodes was noted with the twice-daily premixed insulin versus once- 
daily IGlar (approx. 50\% fewer events in the once-daily IGlar group vs. the premixed insulin group) in persons with T2DM who were inadequately controlled on OADs (mean 4.07 vs. $9.87 /$ person-years; $p<0.0001$ ). In addition, severe hypoglycemia was noted only in the premixed insulin group (0.05/person-year) [20].

In the TTT trial, a randomized, 24-week multicenter trial, the efficacy and associated hypoglycemia risks of IGlar and human NPH insulin added to oral therapy of T2DM were assessed [11]. The trial included 756 overweight men and women with inadequate glycemic control $\left(\mathrm{HbA}_{1 \mathrm{c}}>7.5 \%\right)$ on one or two oral agents. During the study period, the persons received oral agents and bedtime IGlar or NPH insulin once daily. The insulins were titrated using a simple algorithm targeting a FPG level of $\leq 100 \mathrm{mg} / \mathrm{dL}$ $(5.5 \mathrm{mmol} / \mathrm{L})$. The results showed that approximately $60 \%$ of the persons achieved the targeted $\mathrm{HbA}_{1 \mathrm{c}}$ of $\leq 7 \%$ with each insulin type. However, nearly $25 \%$ more persons attained the glycemic target without documented nocturnal hypoglycemia ( $\leq 72 \mathrm{mg} / \mathrm{dL} ; 4.0 \mathrm{mmol} / \mathrm{L}$ ) with the use of IGlar (33.2 vs. $26.7 \% ; p<0.05$ ). Also, the rates of other categories of symptomatic hypoglycemia were $21-48 \%$ lower with IGlar than with NPH insulin [11].

The results of these trials reinforce the safety of basal insulin as compared to premix insulins. The findings also highlight the similar efficacy between basal and premix insulins.

\section{FIRST-GENERATION VERSUS SECOND-GENERATION BASAL INSULIN FOR TITRATION (GLARGINE-300 VS. GLARGINE-100 FOR TITRATION)}

Compared to NPH insulins, long-acting basal insulins represent a remarkable clinical advancement in the management of T2DM, with their longer duration of action, flatter action profiles, and less day-to-day variability with lower risk for hypoglycemia. The TTT concept can be implemented more easily with long-acting basal insulin analogs with less hypoglycemic events. Long-acting second- generation basal insulin analogs, such as insulin degludec 100 units/mL (IDeg-100) and IGlar 300 units/mL (Gla-300) have been developed to further improve the pharmacokinetic/pharmacodynamic (PK/PD) profiles. Second-generation basal insulins have smoother PK/PD profiles versus Gla-100 with lower variability. The EDITION clinical trial development programs for Gla-300 demonstrated that Gla-300 has similar $\mathrm{HbA}_{1 \mathrm{c}}$ reductions as Gla-100 in persons with T2DM but with less hypoglycemia [21].

In the EDITION 2 study, a multicenter openlabel study, the safety and efficacy of newly developed Gla-300 was compared with Gla-100 in persons with T2DM on basal insulin plus OADs [22]. The study assessed the change in $\mathrm{HbA}_{1 \mathrm{c}}$ and the occurrence of hypoglycemic events. It was noted that Gla-300 and Gla-100 exerted similar glycemic control in persons with T2DM using basal insulin in combination with OADs. The glycemic changes observed during the EDITION 2 study were similar to those reported for the EDITION 1 study, which compared Gla-300 and Gla-100 in people with T2DM using a basal bolus insulin treatment [22].

The authors of the study also noted that Gla300 resulted in a significant $23 \%$ reduction in the risk of at least one nocturnal confirmed or severe hypoglycemic event from week 9 to the end of treatment $(p=0.038)$. The reductions noted in nocturnal hypoglycemia with the use of Gla-300 were consistent throughout the study period. The reduction in nocturnal hypoglycemia was predominant during the first 8 weeks of study treatment, corresponding to the titration period of basal insulin. The reduction in nocturnal hypoglycemia observed during the period of titration is of clinical relevance as it may confer optimal titration and, thereby, effective glycemic control with less fear of nocturnal hypoglycemia [22].

\section{COMPARABLE OUTCOMES WITH DIFFERENT TITRATION ALGORITHMS FOR GLA-300}

The aim of the TITRATION study was to evaluate and compare glycemic control, risk of 
hypoglycemia, and change in body weight during Gla-300 titration in the EDITION $(n=104)$ and INSIGHT $(n=108)$ algorithm groups [23] In this study, insulin dosage was increased by 1 unit/day in participants of the INSIGHT algorithm group and the dose was titrated at least once weekly, but not more often than every 3 days, which was the algorithm used by participants in EDITION algorithm group. The target fasting self-monitored blood glucose level was in the range of $80-100 \mathrm{mg} / \mathrm{dL}$. The authors noted that at week 12, 26.9\% (INSIGHT) and $28.8 \%$ (EDITION) of participants achieved a glycated hemoglobin value of $\leq 7 \%$. Participants in both arms of the study were much more satisfied with their new treatment as assessed by the Diabetes Treatment Satisfaction Questionnaire. Most HCPs (86\%) preferred the INSIGHT over the EDITION algorithm [23].

\section{MITIGATING THE RISK OF HYPOGLYCEMIA FOLLOWING TITRATION WITH SECOND- GENERATION BASAL INSULINS}

Following titration, the risk of hypoglycemia can be mitigated by the use of A second-generation basal insulin, such as Gla-300.

The BRIGHT study was the first head-to-head trial that investigated the clinical efficacy and safety of Gla-300 and IDeg-100, two secondgeneration, longer-acting basal insulin analogs [21]. The study included insulin-naive persons with uncontrolled long-standing T2DM who were receiving multiple oral antihyperglycemic drugs. Persons with T2DM were randomized 1:1 to receive evening dosing with Gla-300 $(n=466)$ or IDeg-100 $(n=463)$, titrated to a self-monitored FPG of $80-100 \mathrm{mg} / \mathrm{dL}$. The primary endpoint was $\mathrm{HbA}_{1 \mathrm{c}}$ change from baseline to week 24 . Safety endpoints assessed during the study included incidence and event rates of hypoglycemia [21].

The results of the BRIGHT study noted that Gla-300 was similar to IDeg-100 in terms of $\mathrm{HbA}_{1 \mathrm{C}}$ reduction (from an overall mean $8.6 \%$ at baseline to $7.0 \%$ at week 24 ). Similar proportions of study subjects in the Gla-300 and IDeg-
100 groups achieved an $\mathrm{HbA}_{1 \mathrm{c}}$ target of $<7.0 \%$ without confirmed hypoglycemia $(\leq 70$ and $<54 \mathrm{mg} / \mathrm{dL}$, respectively). Hypoglycemia incidence and event rates over 24 weeks were comparable with both insulins, whereas during the active titration period ( $0-12$ weeks) the incidence and rate of anytime (24-h) confirmed hypoglycemia $\quad(\leq 70 \mathrm{mg} / \mathrm{dL} \quad$ [Gla-300] and $<54 \mathrm{mg} / \mathrm{dL}$ [IDeg-100]) were lower with Gla-300. Most notably, only one severe hypoglycemic event occurred during the entire 24-week trial. The authors of the study concluded that both Gla-300 and IDeg-100 can enable stricter glycemic goals when properly initiated and titrated [21].

In the CONCLUDE (COmparing the efficacy aNd safety of insulin degLUDE and insulin glargine 300 units $/ \mathrm{mL}$ ) head-to-head trial, which involved persons with T2DM inadequately managed with basal insulin and OADs, the primary endpoint assessed was rate of overall symptomatic hypoglycemia in the 36-week maintenance period [24]. The authors reported that the rate of overall symptomatic hypoglycemia during the 36-week maintenance period in persons treated with IDeg was not statistically significantly different from that in persons treated with Gla-300. Since the study could not meet its primary endpoint, the secondary endpoints were considered exploratory and not conclusive [24].

Key Recommendations for Choosing Basal Insulin Profile for Titration

Titrating with basal insulin is easier than using premix insulins for most persons withT2DM in India

The flexibility offered by second-generation basal insulins in terms of dosing time helps in effective titration, with reduced risk of hypoglycemia being a major advantage of these products

\section{CONCLUSION}

Therapeutic inertia is a global concern that impedes the achievement of glycemic control, particularly in persons requiring insulin 
therapy. In real-world practice, titration algorithms exist; however, physicians and persons with T2DM are reluctant to use these algorithms as they perceive them to be very cumbersome. For achieving effective glycemic targets, insulin should be adequately titrated using simple recommendations, and second-generation basal insulins, such as Gla-300, should be used for adequate titration of insulin doses.

\section{ACKNOWLEDGEMENTS}

Sanofi India helped in the organization and logistic support for this expert forum meeting. The content published herein represents the views and opinions of the various contributing authors and does not necessarily represent the views or opinion of Sanofi and/or its affiliates. The details published herein are intended for informational, educational, academic, and/or research purposes and are not intended to substitute for professional medical advice, diagnosis, or treatment.

Funding. This expert opinion initiative and the Rapid Service Fee were funded by Sanofi India.

Medical Writing and Editorial Assistance. Medical writing and editorial support was provided by Dr Rajshri Mallabadi and Dr Kavitha Ganesha from BioQuest Solutions Pvt. Ltd. and paid for by Sanofi, India.

Authorship. All named authors meet the International Committee of Medical Journal Editors (ICMJE) criteria for authorship for this article, take responsibility for the integrity of the work as a whole, and have given their approval for this version to be published.

Disclosures. Sunil M. Jain, Krishna Seshadri, A. G. Unnikrishnan, Manoj Chawla, Pramila Kalra, V. P. Vipin, E. Ravishankar, Jay Chordia, Sambit Das, Jasjeet Wasir, S. M. Bandookwala, Neelakshi Deka, Ghanshyam Agarwal, G. Vijaykumar and Suhas Erande have nothing to disclose.
Compliance with Ethics Guidelines. This article is based on previously conducted studies and does not contain any studies with human participants or animals performed by any of the authors.

Open Access. This article is distributed under the terms of the Creative Commons Attribution-NonCommercial 4.0 International License (http://creativecommons.org/licenses/ by-nc/4.0/), which permits any noncommercial use, distribution, and reproduction in any medium, provided you give appropriate credit to the original author(s) and the source, provide a link to the Creative Commons license, and indicate if changes were made.

\section{REFERENCES}

1. Berard L, Bonne Maire M, Mical M, et al. Insights into optimal basal insulin titration in type 2 diabetes: results of a quantitative survey. Diabetes Obes Metab. 2018;20(2):301-8.

2. Russel Jones D, Pouwer F, Khunti K. Identification of barriers to insulin therapy and approaches to overcoming them. Diabetes Obes Metab. 2018;20(3):488-96.

3. Arnolds $\mathrm{S}$, Heise $\mathrm{T}$, Flacke $\mathrm{F}$, et al. Common standards of basal insulin titration in type 2 diabetes. J Diabetes Sci Technol. 2013;7(3):771-88.

4. Kirk JK, Stegner J. Self-monitoring of blood glucose: practical aspects. J Diabetes Sci Technol. 2010;4(2): 435-9.

5. Ong WM, Chua SS, Ng CJ. Barriers and facilitators to self-monitoring of blood glucose in people with type 2 diabetes using insulin: a qualitative study. Patient Prefer Adherence. 2014;8:237-46.

6. Owens DR, Traylor L, Dain MP, et al. Efficacy and safety of basal insulin glargine 12 and 24 weeks after initiation in persons with type 2 diabetes: a pooled analysis of data from treatment arms of 15 treat-to-target randomised controlled trials. Diabetes Res Clin Pract. 2014;106(2):264-74.

7. Polonsky WH, Arsenault J, Fisher L, et al. Initiating insulin: How to help people with type 2 diabetes start and continue insulin successfully. Int J Clin Pract. 2017;71:8. 
8. Gerstein HC, Yale JF, Harris SB, et al. A randomized trial of adding insulin glargine vs. avoidance of insulin in people with Type 2 diabetes on either no oral glucose-lowering agents or submaximal doses of metformin and/or sulphonylureas: the Canadian INSIGHT (Implementing New Strategies with Insulin Glargine for Hyperglycaemia Treatment) study. Diabet Med. 2006;23(7):736-42.

9. Yki-Jarvinen $\mathrm{H}$, Kauppinen-Makelin $\mathrm{R}$, Tiikkainen $\mathrm{M}$, et al. Insulin glargine or NPH combined with metformin in type 2 diabetes: the LANMET study. Diabetologia. 2006;49(3):442-51.

10. Davies M, Storms F, Shutler S, et al. Improvement of glycemic control in subjects with poorly controlled type 2 diabetes: comparison of two treatment algorithms using insulin glargine. Diabetes Care. 2005;28(6):1282-8.

11. Riddle MC, Rosenstock J, Gerich J. The treat-totarget trial: randomized addition of glargine or human NPH insulin to oral therapy of type 2 diabetic patients. Diabetes Care. 2003;26(11):3080-6.

12. Davidson MB. Insulin therapy: a personal approach. Clin Diabetes. 2015;33(3):123-35.

13. Garg SK, Admane K, Freemantle N, et al. Patient-led versus physician-led titration of insulin glargine in patients with uncontrolled type 2 diabetes: a randomized multinational ATLAS study. Endocr Pract. 2015;21(2):143-57.

14. Harris S, Yale JF, Dempsey E, et al. Can family physicians help patients initiate basal insulin therapy successfully?: randomized trial of patient-titrated insulin glargine compared with standard oral therapy: lessons for family practice from the Canadian INSIGHT trial. Can Fam Physician. 2008;54(4):550-8.

15. Yki-Jarvinen $\mathrm{H}$, Juurinen $\mathrm{L}$, Alvarsson $\mathrm{M}$, et al. INITIATE (INITiate Insulin by Aggressive Titration and Education): a randomized study to compare initiation of insulin combination therapy in type 2 diabetic patients individually and in groups. Diabetes Care. 2007;30(6):1364-9.

16. Meneghini L, Koenen C, Weng W, et al. The usage of a simplified self-titration dosing guideline (303 Algorithm) for insulin detemir in patients with type 2 diabetes-results of the randomized, controlled
PREDICTIVE 303 study. Diabetes Obes Metab. 2007;9(6):902-13.

17. Deerochanawong C, Bajpai S, Dwipayana IMP, et al. Optimizing glycemic control through titration of insulin glargine $100 \mathrm{U} / \mathrm{mL}$ : a review of current and future approaches with a focus on Asian populations. Diabetes Ther. 2017;8(6):1197-214.

18. Mauricio D, Meneghini L, Seufert J, et al. Glycaemic control and hypoglycaemia burden in patients with type 2 diabetes initiating basal insulin in Europe and the USA. Diabetes Obes Metab. 2017;19(8): 1155-64.

19. Raskin $\mathrm{P}$, Allen E, Hollander $\mathrm{P}$, et al. Initiating insulin therapy in type 2 diabetes: a comparison of biphasic and basal insulin analogs. Diabetes Care. 2005;28(2):260-5.

20. Janka HU, Plewe G, Riddle MC, et al. Comparison of basal insulin added to oral agents versus twice-daily premixed insulin as initial insulin therapy for type 2 diabetes. Diabetes Care. 2005;28(2):254-9.

21. Rosenstock J, Cheng A, Ritzel R, et al. More similarities than differences testing insulin glargine 300 units/ml versus insulin degludec 100 units/mL in insulin-naive type 2 diabetes: the randomized headto-head BRIGHT trial. Diabetes Care. 2018;41(10): 2147-54.

22. Yki-Järvinen $H$, Bergenstal R, Ziemen $M$, et al. New insulin glargine 300 units/mL versus glargine 100 units/mL in people with type 2 diabetes using oral agents and basal insulin: glucose control and hypoglycemia in a 6-month randomized controlled trial (EDITION 2). Diabetes Care. 2014;37(12): $3235-43$.

23. Yale JF, Berard L, Groleau M, et al. TITRATION: a randomized study to assess 2 treatment algorithms with new insulin glargine 300 units/mL. Can J Diabetes. 2017;41(5):478-84.

24. Philis-Tsimikas A, Klonoff DC, Khunti K, et al. CONCLUDE: a trial COmparing the efficacy aNd safety of insulin degLUDEc and insulin glargine 300 units/mL in subjects with type 2 diabetes mellitus inadequately treated with basal insulin and oral antidiabetic drugs. Symposium at the 55th Annual Meeting of the European Association for the Study of Diabetes (EASD), Barcelona, Spain; 16-20 September 2019. 